\title{
Pattern-specific expression of the Drosophila decapentaplegic gene in imaginal disks is regulated by $3^{\prime}$ cis- regulatory elements
}

\author{
James D. Masucci, Rosalynn J. Miltenberger, and F. Michael Hoffmann ${ }^{1}$ \\ McArdle Laboratory for Cancer Research, University of Wisconsin, Madison, Wisconsin 53706 USA
}

\begin{abstract}
The pattern of structures on most of the adult cuticle of Drosophila is determined in the larval imaginal disks. The Drosophila growth factor homolog decapentaplegic (dpp) is believed to participate in pattern formation in imaginal disks, primarily along what will become the proximal-to-distal axis of adult appendages. We report that $d p p$ expression in wing, leg, and eye-antennal imaginal disks is localized to a band of cells along the presumptive proximal-to-distal axis. The pattern and level of $d p p$ expression in imaginal disks is affected by mutant lesions that remove 3 ' cis-regulatory sequences. We demonstrate that one portion of the 3 ' cisregulatory region contains regulatory elements sufficient to activate gene expression in a subset of the cells that normally express $d p p$ in the imaginal disks, allowing rescue of $d p p$ mutant phenotypes. We propose that the complete $d p p$ expression pattern is generated by an array of 3 ' regulatory elements that differ in their potency in specific disks and in certain positions within a disk. The identification of the factors that activate these elements should provide clues as to how positional information is encoded in imaginal disks.
\end{abstract}

[Key Words: Drosophila; pattern formation; imaginal disks; gene expression]

Received June 11, 1990; revised version accepted August 15, 1990.

With the exception of the abdominal cuticle, the entire Drosophila adult cuticle is derived from the 19 imaginal disks. The imaginal disks are folded sheets of epithelial cells that each arise from a cluster of 2-20 cells allocated in the embryo at blastoderm formation (for review, see Postlethwait 1978). These cells undergo at least one mitotic division between 3 and $10 \mathrm{hr}$ after oviposition (Wieschaus and Gehring 1976) and then remain quiescent until late in the first-larval instar or early in the second-larval instar. At this time, rapid cell divisions ensue until the mature disks contain 4,000-50,000 cells late in the third-larval instar. At the onset of metamorphosis, the disks respond to increasing titers of 20-hydroxy ecdysone, resulting in a series of cell rearrangements and shape changes that enable the epithelial sheet to unfold, secrete cuticle, and form the adult appendages (Fristrom 1976).

The pattern of structures on the Drosophila adult cuticle is established in the larval imaginal disks by a molecularly undefined system of positional information. The generation of imaginal disk fate maps indicates that at the third-larval instar imaginal disk cells are determined to become specific structures on the adult cuticle (for review, see Bryant 1978). Analysis of marked cell clones indicates that cell lineage is not the determining factor in imaginal disk pattern formation. Experiments

${ }^{1}$ Corresponding author. on the regenerative properties of imaginal disk fragments led to proposals that systems of positional information or positional coordinates within imaginal disks specify the fate of cells (for review, see Meinhardt 1982).

Genes that participate in specifying cell fates in imaginal disks may be expected to exhibit spatially restricted patterns of expression in imaginal disks and, when mutated, to cause a loss or duplication of imaginal disk-derived structures. Several genes are known to have spatially restricted patterns in the imaginal disks including the homeotic genes Ultrabithorax, Antennapedia, engrailed (en), the integrin homolog PS2, and the secreted proto-oncogene homolog wingless (Brower et al. 1984; Kornberg et al. 1985; White and Wilcox 1985; Wirz et al. 1985; Baker 1988). One class of mutant alleles of the decapentaplegic $(d p p)$ gene causes phenotypic defects in imaginal disk-derived structures (for reviews, see Gelbart 1989; Hoffmann 1990). The product of $d p p$ is homologous in sequence and biochemical properties to the secreted proteins in the transforming growth factor$\beta$ (TGF- $\beta$ ) family of growth factors (Padgett et al. 1987; Panganiban et al. 1990). In agreement with the biochemical characterization of $d p p, d p p$ function in the wing imaginal disk is cell nonautonomous, that is, the phenotype of cells mutant for $d p p$ is rescued by $d p p$-expressing cells elsewhere in the same wing imaginal disk (Spencer 1984; Posakony 1987; Posakony et al. 1990).

Although null mutations at $d p p$ are embryonic lethals 
causing defective gastrulation and dorsal-ventral polarity (Irish and Gelbart 1987), the $30 d p p^{\text {disk }}$ alleles result in the absence of adult cuticular structures along the proximal-to-distal axis of adult appendages (Spencer et al. 1982). The $d p p^{d i s k}$ alleles map from 2 to $26 \mathrm{~kb}, 3^{\prime}$ of the transcribed region of $d p p$, indicating the presence of cis-regulatory sequences required for imaginal disk expression of $d p p$ (St. Johnston et al. 1990). With the exception of two internal deletions of the $d p p$ disk region, all of the $d p p^{d i s k}$ alleles are chromosomal rearrangements. The mildest alleles map farthest from the transcribed region of $d p p$ and result in the absence of structures on either the wing blade or the eye. More severe alleles map closer to the transcribed region and affect more structures derived from all of the imaginal disks. The effects of the $d p p^{\text {disk }}$ mutations can be seen in the imaginal disks, which are smaller and show signs of defective cell proliferation and extensive cell death (Bryant 1988).

To understand the functions of the $3^{\prime}$ regulatory region in directing $d p p$ expression in imaginal disks, we have determined the patterns of wild-type $d p p$ expression in the disks from third-instar larvae and early pupae, as well as $d p p$ expression in disks homozygous for mild $d p p$ mutations. We demonstrate that $d p p$ is expressed in cells just anterior to the anterior-posterior compartment boundary in the wing and leg imaginal disks, in the morphogenetic furrow and periphery of the eye disk, and in a medial-to-lateral stripe of the antennal disk. Upon evagination of the disks, this expression follows the proximal-to-distal axis of adult appendages. Mutations reduce expression in specific disks and in specific regions of the disks. We provide evidence that the $3^{\prime}$ regulatory sequences induce pattern-specific expression from either a $d p p$ promoter or a heterologous promoter. The expression of $d p p$ from the transposon construct is sufficient to rescue the $d p p$ mutant phenotypes.

\section{Results}

Wild-type pattern of imaginal disk dpp expression

Digoxigenin-labeled DNA probes were used to detect dpp mRNA in whole-mount imaginal disks of third-instar larvae. $d p p$ mRNA was detected in specific subsets of imaginal disk cells (Fig. 1). The earliest disks examined were from animals at the early third-instar larval stage (Fig. la,d,g). Detectable $d p p$ expression is restricted to the primordia of the ventral aspect of the wing and the medial side of the leg (Fig. 1a,d, arrows). As the disks mature, the number of cells expressing dpp mRNA increases to form a discontinuous stripe across the wing and leg imaginal disks (Fig. 1b,e). Late in the third-larval instar, another stripe of staining is detected along the posterior edge of the wing disk (Fig. 1c, arrowhead). In contrast to the band of expression across the wing disk, $d p p$ expression in the leg disk is more intense in the region that becomes the lateral side of the leg (Fig. 1f, ls).

In the early third-instar eye imaginal disk, the stripe of $d p p$-expressing cells extends around the periphery of the eye disk primordia (Fig. 1g). As the disk matures, the stripe of $d p p$ expression along the posterior portion of the disk migrates with the morphogenetic furrow while the lateral and medial stripes of expression remain stationary (Fig. lh). As in the leg imaginal disk, the stripe of cells expressing $d p p$ on the lateral edge of the eye disk is more intense than that on the medial edge (Fig. 1i). The cells expressing $d p p$ in the early third-instar antennal disk are situated in the centrolateral portion of the disk (Fig. $1 \mathrm{~g}$, arrow). It becomes apparent in older disks that this expression is on the lateral side of the presumptive antennal segments. Also at this stage, faint expression is detected across the medial edge of each antennal segment. In late third-instar disks, two additional stripes are detected in the antennal disk (Fig. 1i, arrowheads).

\section{dpp expression is adjacent to the compartment boundary}

Expression of the segment polarity gene en is localized to the posterior compartments of the leg and wing imaginal disks (Kornberg et al. 1985). Digoxigenin-labeled probes for $d p p$ (Fig. 2c,f) and en (Fig. 2a,d) were used separately and in the same disks (Fig. 2b,e) to compare the location of $d p p$-expressing cells with respect to the anterior-posterior compartment boundary defined by en expression. The results indicate that $d p p$ is expressed in cells just anterior to the cells expressing en. This experiment does not rule out that some cells at the boundary coexpress $d p p$ and $e$. Consistent with these localization results, analysis of $d p p$ mutant clones in wing disks has led to the conclusion that expression of $d p p$ anterior to the compartment boundary is critical for $d p p$ function in the disk (Posakony 1987; Posakony et al. 1990).

dpp expression persists during the initial stages of disk eversion

At the onset of metamorphosis, the wing and leg imaginal disks evaginate and take on the shape of the adult wing and leg appendages while the two sets of eye-antennal disks fuse to form the head capsule. The localization of $d p p$ mRNA was determined in imaginal disks from white prepupae (Fig. $3 \mathrm{a}, \mathrm{d}, \mathrm{g}$ ), and prepupae at $3.5 \mathrm{hr}$ (Fig. $3 \mathrm{~b}, \mathrm{e}, \mathrm{h}$ ) and $5.5 \mathrm{hr}$ (Fig. $3 \mathrm{c}, \mathrm{f}, \mathrm{i})$ postpuparium formation. Staining seen in the mature third-instar disks persists through the initial stages of eversion. Additional expression of $d p p$ is detected in the anterior region of the wing pouch of white prepupae (Fig. $3 \mathrm{a}$, as). In the everting wing and leg imaginal disks (Fig. $3 \mathrm{c}, \mathrm{f}$ ), the previously discontinuous spots of expression detected in the third-instar imaginal disks (Fig. 1c,f) are revealed as continuous stripes along the proximal-distal axis. The expressing cells are present on both the dorsal and ventral surfaces of the wing blade; however, in everting leg disks, the lateral stripe of expression (Fig. 3f, ls) is more intense than the medial stripe (Fig. 3f, ms).

All expression detected in the eye-antennal disks prior to puparium formation (Fig. $3 \mathrm{~g}$ ) persists as the two sets of disks fuse (Fig. 3h,i). The anterior-most cells of the eye, which are the last cells to enter the morphoge- 

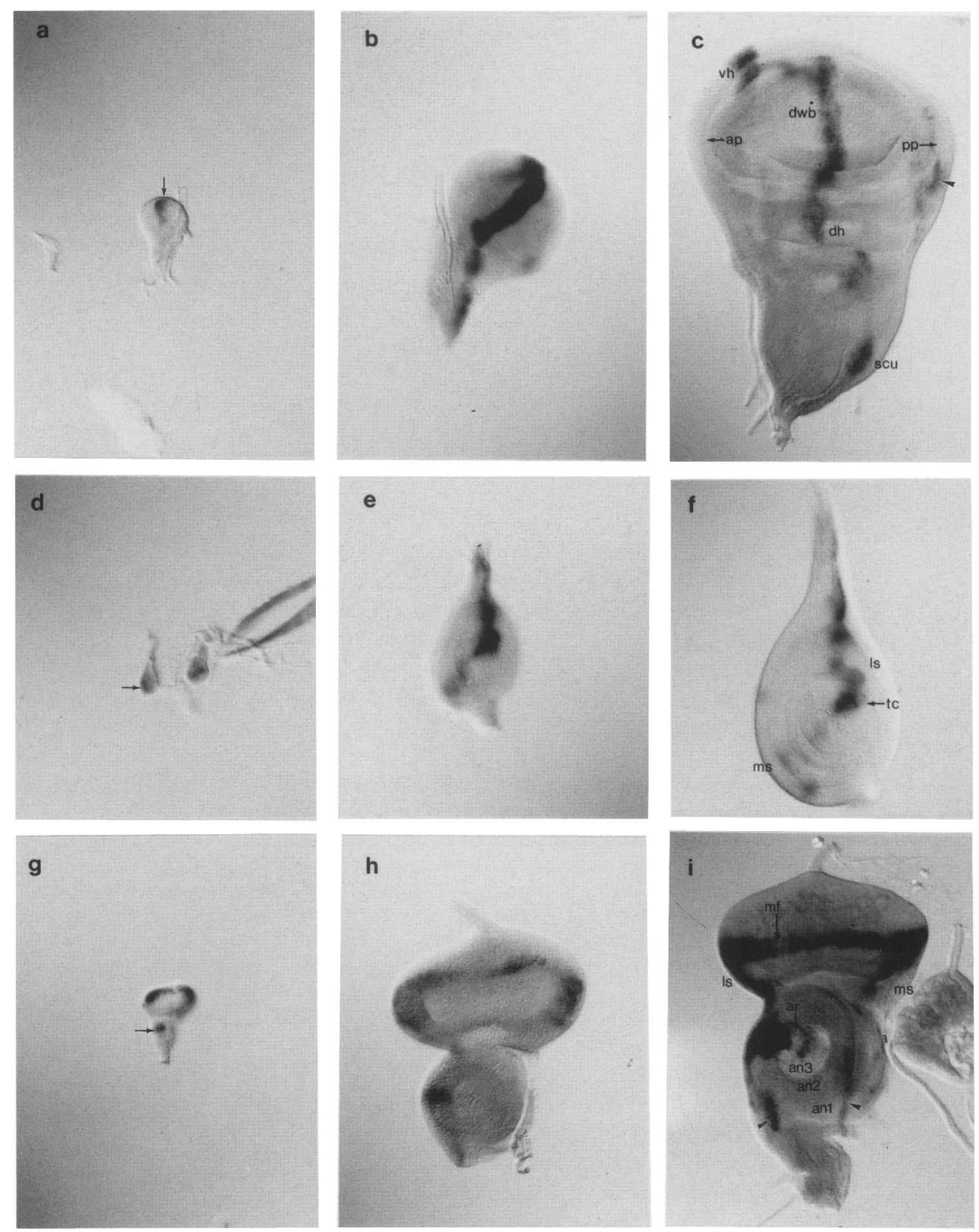

Figure 1. Wild-type $d p p$ expression in imaginal disks at three stages of the developing third-larval instar. Imaginal disks from early (left), intermediate (center), and late (right) third-instar larvae were analyzed for expression of $d p p$ RNA. (a-c) Developing wing disks are oriented anterior to the left and ventral to the top. $d p p$ expression at early third instar is restricted to the ventral-most region of the wing disk $(a$, arrow). Later, dpp expression extends discontinuously across the center of the entire wing disk and appears at the posterior edge ( $c$, arrowhead). Regions of the disk are indicated as follows: (ap) anterior border of wing pouch; (pp) posterior border of wing pouch; (dwb) distal tip of wing blade; (vh) ventral hinge structures; (dh) dorsal hinge structures; (scu) scutellum. (d-f) Developing leg disks are oriented anterior to the left and lateral side to the top. dpp expression at early third instar is restricted to the medial side of the leg disk $(d$, arrow). The expression evolves into a discontinuous stripe across the entire disk $(e, f)$. The lateral stripe of $d p p$ expression $(1 \mathrm{~s}$ in $f)$ is more prominent than the medial stripe $(\mathrm{ms}$ in $f)$. Fate map position of tarsal claws $(\mathrm{tc})$ is indicated. $(g-i)$ Developing eye-antennal disks are oriented lateral side to the left and posterior to the top. Eye primordia is on top; antennal primordia is at bottom. $d p p$ expression in the early third-instar eye disk surrounds the periphery $(g)$ and, in the antennal portion of the disk, is localized to the lateral side of the disk $(g$, arrow $)$. The posterior limit of eye disk expression travels with the morphogenic furrow ( $\mathrm{mf}$ in i). Lateral and medial stripes of eye disk expression (ls and $\mathrm{ms}$ in i) extend anteriorly into the antennal disk. $d p p$ expression on the lateral side of the antennal disk is maintained in later disks $(h, i)$. Additional $d p p$ expression is detected in late third-instar antennal disks in two stripes ( $i$, arrowheads) and across the medial side of the antennal segments. Regions of the disk are indicated as follows: (ar) aristae; (an3) third antennal segment; (an2) second antennal segment; (an 1) first antennal segment. 

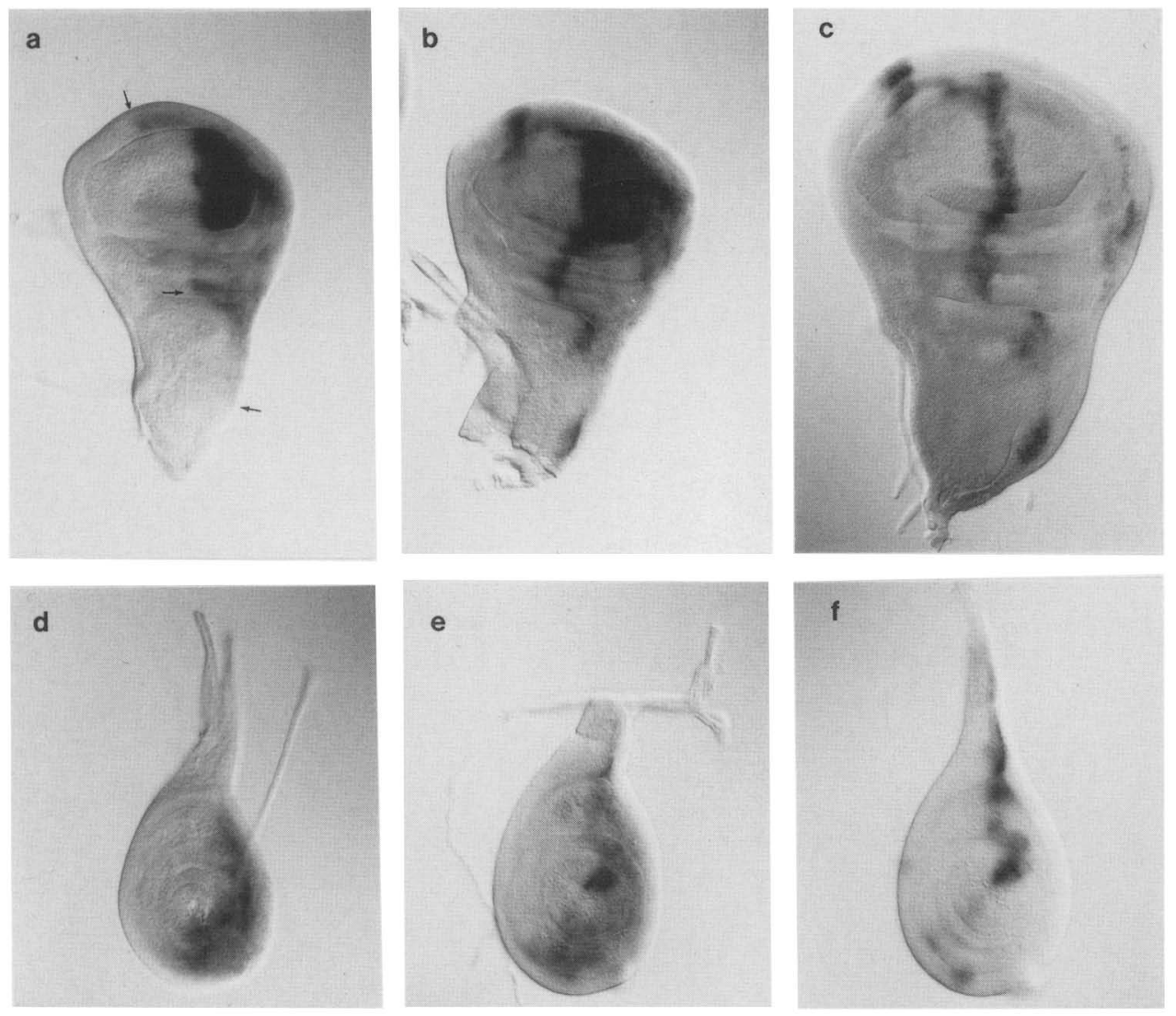

Figure 2. Comparison of $d p p$ and en patterns of expression in late third-instar wing and leg imaginal disks. Orientations are as in Fig. 1. Probes for $e n$ alone (left), both $d p p$ and en (center), or $d p p$ alone (right) detect expression in wing $(a-c)$ and leg $(d-f)$ imaginal disks of wild-type larvae. $(a, d)$ Wild-type en staining shows that en is expressed in the entire posterior compartment of wing and leg disks. Arrows in $a$ indicate the anterior-most limits of the en expression domain. $(b, e)$ The staining pattern with both probes is the sum of the patterns observed in $a, d$ and $c, f$, indicating that $d p p$ is expressed anterior and adjacent to $e n$. $(c, f)$ The wild-type $d p p$ staining pattern is shown.

netic furrow, continue expressing $d p p$ after puparium formation. The result is a ring of expression around the border of the developing eye. Furthermore, cells that express $d p p$ in the lateral (Fig. 3g, ls) and medial stripes (Fig. $3 \mathrm{~g}, \mathrm{~ms}$ ) of the eye in late third-instar disks continue to express $d p p$, although the medial stripe broadens by $3.5 \mathrm{hr}$ postpurarium formation (Fig. $3 \mathrm{~h}$ ). The cells expressing $d p p$ in the everting antennae form a continuous stripe along the lateral side of the antennae (Fig. 3i, arrowhead). The faint medial stripe is not detected in the everting antennal disk.

\section{dpp mutations decrease dpp mRNA expression}

Mutations that specifically affect $d p p$ function in the imaginal disks map in a $3^{\prime}$-nontranscribed region proposed to contain cis-regulatory sequences (St. Johnston et al. 1990). Most of these mutations are chromosomal rearrangements that break the DNA in the $3^{\prime}$ regulatory region and separate the putative regulatory sequences on the proximal side of the break from the $d p p$ transcriptional unit on the distal side of the break. Because the imaginal disks from larvae mutant for strong $d p p^{\text {disk }}$ al- leles are too small to be readily dissected, imaginal disks from larvae homozygous for three of the mildest $d p p^{d i s k}$ mutations, $d p p^{d-h o}, d p p^{d 5}$, or $d p p^{d-b l k}$, were probed with digoxigenin-labeled DNA probes. The mutation $d p p^{d-h o}$ is a 3 -kb deletion of sequences $23 \mathrm{~kb} \mathrm{3}$ of the $d p p$ transcriptional unit (Blackman et al. 1987). $d p p^{d-h o}$ causes a held-out wing and defects in a sensory structure on the wing blade, the sensilla campaniformia 25 (sc25; Spencer et al. 1982). The $d p p^{d s}$ mutation is a DNA rearrangement with one breakpoint $21 \mathrm{~kb} 3^{\prime}$ of the $d p p$ transcriptional unit (St. Johnston et al. 1990), which reduces the size of the wing blade and slightly reduces the size of the eye. Both mutations reduce the level of $d p p$ expression in the mutant wing disks (Fig. $4 \mathrm{~b}, \mathrm{c}$ ), as compared to a wild-type disk (Fig. 4a). The area of reduced expression seen in the wing disk of $d p p^{d-h o}$ mutants corresponds to the fate map position of the sc25. $d p p$ expression in the $d p p^{d 5}$ mutant wing disk is reduced along the entire anterior-posterior compartment boundary, resulting in only five localized spots of expression rather than the discontinuous stripe of $d p p$ expression observed in wild-type disks. In $d p p^{d 5}$ eye disks, which form a slightly reduced eye, the level of $d p p$ expression along the morphogenetic 


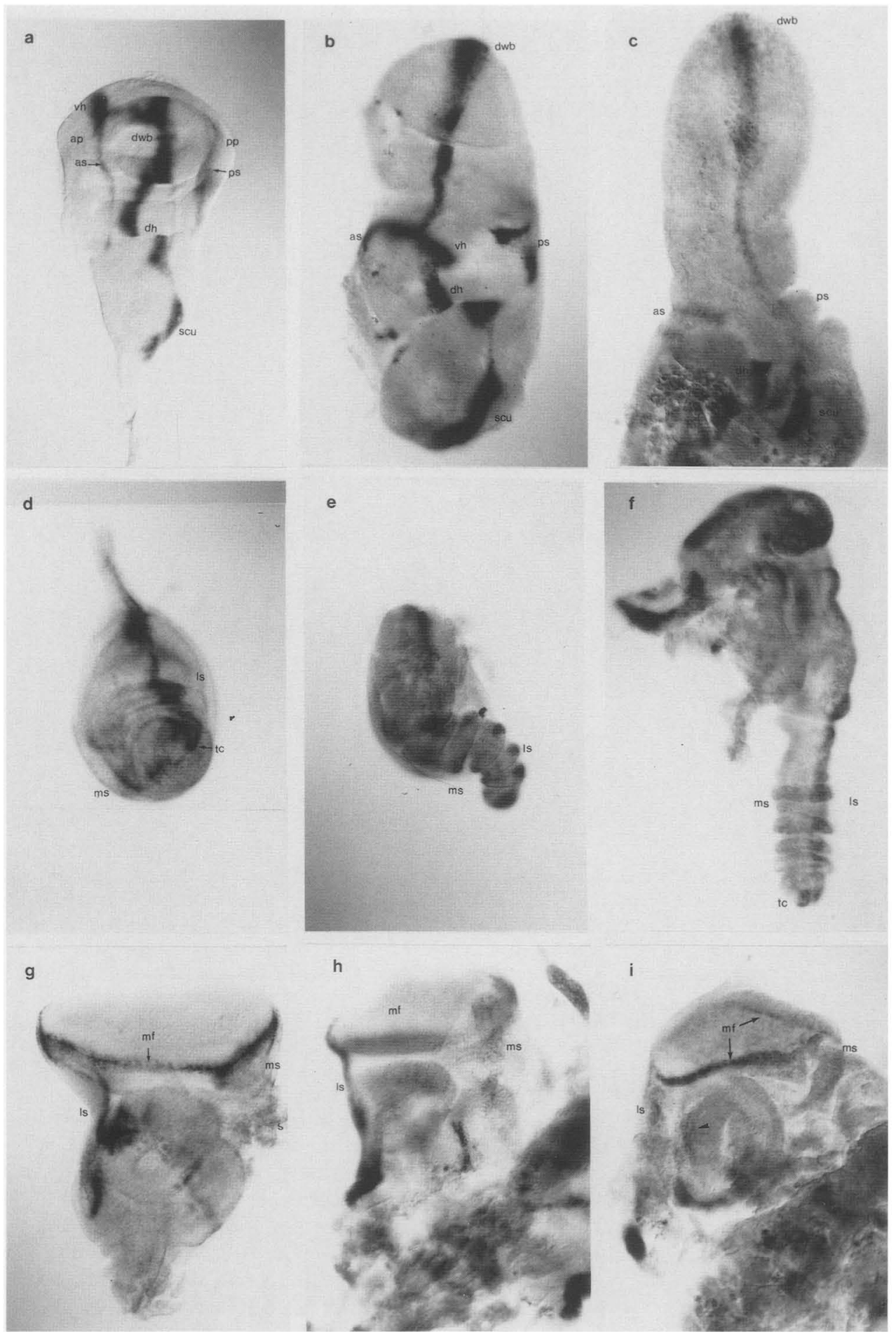

Figure 3. Wild-type $d p p$ expression during imaginal disk eversion. Orientations and abbreviations are as in Fig. 1. Everting wing $(a-c)$, leg $(d-f)$, and eye-antennal $(g-i)$ disks from white prepupae (left), animals $3.5 \mathrm{hr}$ postpuparium formation (center), and animals $5.5 \mathrm{hr}$ postpuparium formation (right) were analyzed for expression of $d p p$ RNA. Comparison of the patterns of expression in disks beginning to evert $(a, d, g)$ with mature third-instar disks (shown in Fig. $1 \mathrm{c}, \mathrm{f}, \mathrm{i}$ ) indicates that most $d p p$ expression persists unchanged during the initial stages of eversion. During eversion, a novel stripe of expression is detected in the anterior portion of the wing disk (arrow at as). In the everting wing and leg disks $(b, c$ and $e, f)$ the stripe of $d p p$ expression in the disk is resolved into a band of expression along the proximal-to-distal axis of the appendages. The distal end of the wing blade is up in $b$ and $c$, and the distal end of the leg is down in $e$ and $f$. Two sets of eye-antennal disks fuse along their medial sides to form the head capsule $(h, i)$. Antennae are everting into the plane of the page (for further details, see text). 

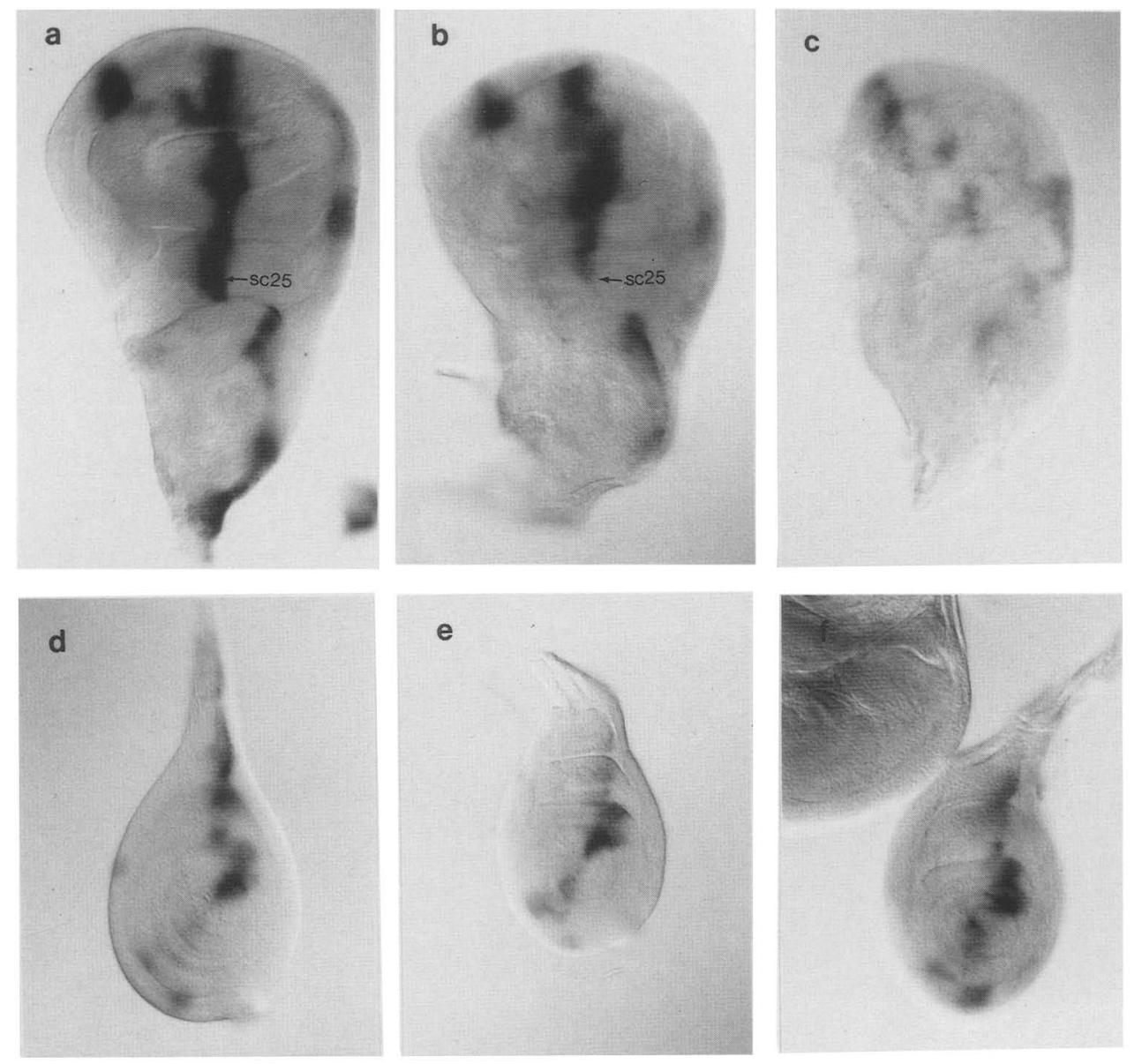

Figure 4. $d p p$ expression in mild $d p p^{d i s k}$ mutants that specifically affects wing development. Orientations are as in Fig. 1. Imaginal disks from late third-instar larvae, either wild type (left) or homozygous for the mutations $d p p^{d-h o}$ (center) or $d p p^{d 5}$ (right), were analyzed for $d p p$ RNA expression. Disks were processed in parallel. When the wild-type pattern of $d p p$ expression in the wing disk (a) is compared to the $d p p$ expression in a $d p p^{d-h o}$ mutant wing disk $(b \mid$, a reduction in expression is apparent in the dorsal hinge area of the mutant disk. This is the same region of the wing disk that gives rise to the sensilla campaniforme 25 (sc25 in $a, b$ ), the structure that is missing in $d p p^{d-h o}$ mutant wings. $(c) d p p^{d 5}$ substantially reduces the intensity of $d p p$ expression across the mutant wing disk such that only a subset of the wild-type pattern is detected. $(d-f)$ Neither mutation affects $d p p$ expression in the leg disks.

furrow is reduced but detectable (not shown). Consistent with the normal development of the leg structures in both mutant backgrounds, $d p p$ expression is unaffected in either $d p p^{d-k o}$ or $d p p^{d 5}$ mutant leg disks (Fig. $4 \mathrm{~d}-\mathrm{f}$ ).

The mutation $d p p^{d-b l k}$ is a $5-\mathrm{kb}$ deletion of sequences $17 \mathrm{~kb} 3^{\prime}$ of the $d p p$ transcribed region (Blackman et al. 1987). $d p p^{d-b l k}$ causes a reduction in the number of eye facets from the wild-type complement of 700 (Fig. 5c) to $\sim 100-200$ (Fig. 5a) but does not affect any other imaginal disk-derived structures. Each ommatidium in the mutant eye exhibits a full complement of photoreceptor cells that are patterned normally. The axis of dorsalventral symmetry that normally bisects the ommatidial clusters (Fig. 5e, arrow) is asymmetrically positioned in the $d p p^{d-b l k}$ eye (Fig. $5 \mathrm{~d}$ ), where all but a few ventral ommatidia are missing. Examination of cell death in the $d p p^{d-b l k}$ mutant eye disks by accumulation of acridine orange indicates that cell death is more prevalent on the ventral side of the mutant eye disk (Fig. 6a, arrow) when compared to a wild-type disk (Fig. $6 \mathrm{~b}$ ). Also apparent in
Figure 6 is the reduced size of the eye disk in $d p p^{d-b l k}$ mutant animals, indicating that decreased cell proliferation and/or increased cell death probably occur throughout the development of the eye disk. $d p p$ expression in the $d p p^{d \cdot b l k}$ mutant eye disks (Fig. 7a) is reduced below the level detectable with a digoxigenin-labeled DNA probe that readily detects $d p p$ RNA in wild-type eye disks (Fig. 1i) and in leg disks (Fig. 7d) derived from $d p p^{d-b l k}$ mutant larvae.

dpp disk region sequences impart imaginal disk expression to the dpp and alcohol dehydrogenase promoters

A 4-kb BamHI fragment spanning 106-110 of the $d p p^{\text {disk }}$ region was inserted into a P-element transposon that contained a $d p p$ promoter and protein-coding exons; the alcohol dehydrogenase $(a d h)$ gene was present on the transposon as a selectable marker (Fig. 8a). The 106-110 BamHI fragment in TnJMB7 is located just $5^{\prime}$ to the $a d h$ 


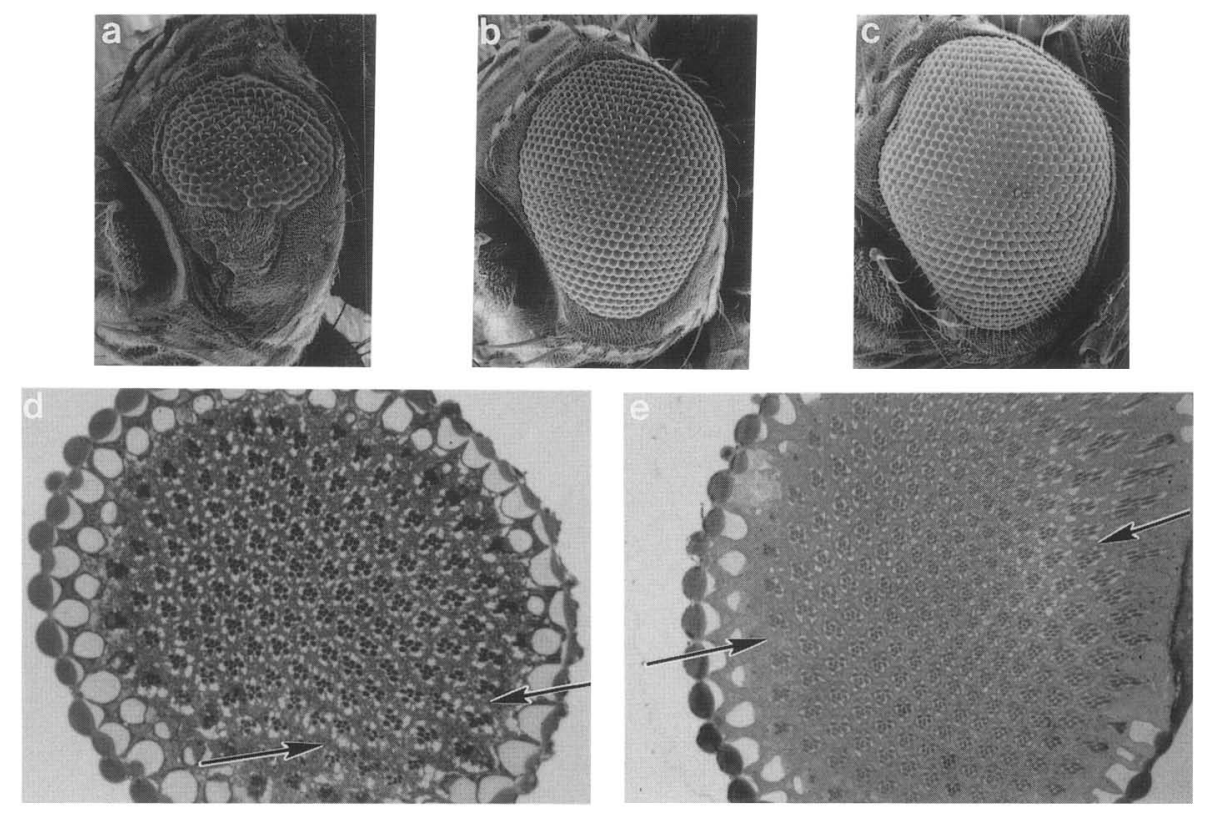

Figure 5. Adult eye phenotypes of $d p p^{d-b l k}$ mutants and transposon-rescued $d p p^{d-b l k}$ mutants. Anterior is to the left and dorsal is to the top. (a) Approximately 100-200 ommatidia develop in the dorsal region of the $d p p^{d-b l k}$ mutant eye. (b) One copy of TnJMB7 rescues the $d p p^{d-b l k}$ mutant eye phenotype to near wild-type number of ommatidia and fully restores a normal, highly ordered array to the ommatidia. (c) A wild-type eye is made up of $\sim 700$ ommatidia. $(d, e)$ Equatorial cross sections of adult eyes reveal the predominant dorsal orientation of ommatidia in $d p p^{d-b l k}$ mutants $(d)$ compared to wild type $(e)$. Each ommatidium contains a full complement of photoreceptor cells, which are organized in a trapezoidal array. The trapezoids point in opposite directions on either side of the equator (arrows), which divides the wild-type eye into equal dorsal and ventral halves. In the $d p p^{d-b l k}$ mutant eye, only a few ventrally oriented ommatidia exist.

promoter. A transgenic line was established with transposon TnJMB7. Digoxigenin-labeled DNA probes for adh were used to determine whether the $4-\mathrm{kb} d p p$ fragment affected the expression of the heterologous adh

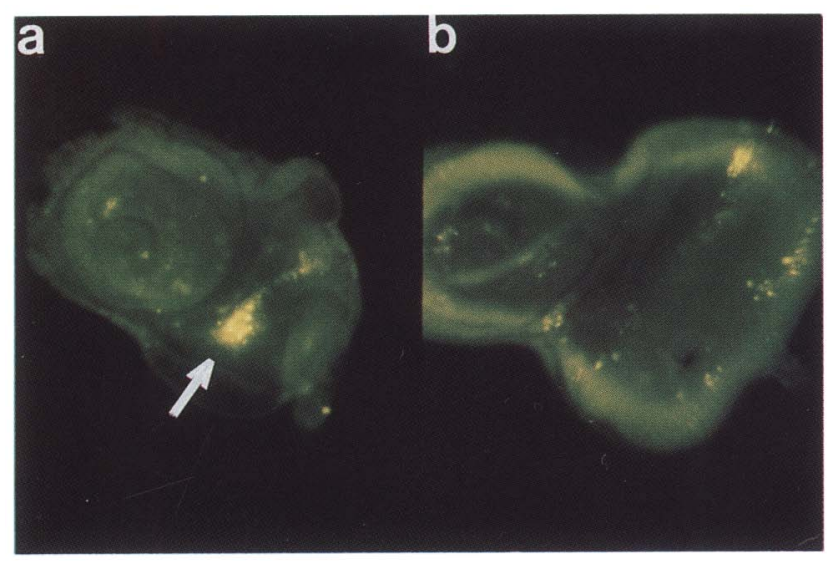

Figure 6. Acridine orange staining of $d p p^{d-b l k}$ mutant and wild-type eye-antennal disks. Disks are oriented anterior left and dorsal to the top. Unfixed, late third-instar eye-antennal disks from $d p p^{d-b l k}$ mutant $(a)$ and wild type $(b)$ were stained with the fluorescent dye acridine orange to mark regions of cell death. $d p p^{d-b l k}$ mutant eye disks are aberrantly shaped and smaller than wild type. A stripe of fluorescence just anterior to the morphogenetic furrow is common to both mutant and wild-type eye disks. In the $d p p^{d-b l k}$ mutant eye disk, a novel patch of intense fluorescence is detected on the ventral side (arrow). promoter. adh mRNA was detectable in cells in the eye disk (Fig. 7c) and also in the wing (not shown) and leg imaginal disks (Fig. $7 \mathfrak{f}$ ) of transgenic animals in patterns similar to parts of the wild-type pattern of $d p p$-expressing cells (Fig. lf,i). adh RNA expressed from an independently derived transposon line of a similar construct (Fig. $8 \mathrm{~b}$ ) was detected in a pattern indistinguishable from that seen with TnIMB7 (data not shown). The identical patterns of adh expression in the disks provided by both transposons argue against the existence of artifactual expression due to site of insertion. No adh mRNA was detected in imaginal disks from control flies that were transgenic for the $d p p \mathrm{P}$ element lacking the disk sequences (data not shown).

A P-element transposon lacking the 106-110 fragment but containing the $d p p$ promoter and proteincoding exons (Hin region) has no effect on $d p p^{\text {disk }}$ mutations (Hoffmann and Goodman 1987). Similarly, a transposon containing only $106-110$ has no effect on $d p p^{\text {disk }}$ mutant phenotypes, indicating a cis-acting mechanism (data not shown). The TnJMB7 transposon was tested for its ability to rescue the $d p p^{d-b l k}$ mutation. One copy of the transposon was sufficient to rescue the phenotype of $d p p^{d-b l k}$ (Fig. 5b) to wild type. Eye disks from $d p p^{d-b l k}$ larvae carrying two copies of TnJMB7 in the genome were examined for $d p p$ expression and found to have detectable $d p p$ expression in a pattern indistinguishable from wild type (Fig. $7 \mathrm{~b}$ ). The eye-specific defects caused by the deletion of the 106-110 region in $d p p^{d-b l k}$ and the rescue of the eye phenotype by TnJMB7 are consistent 


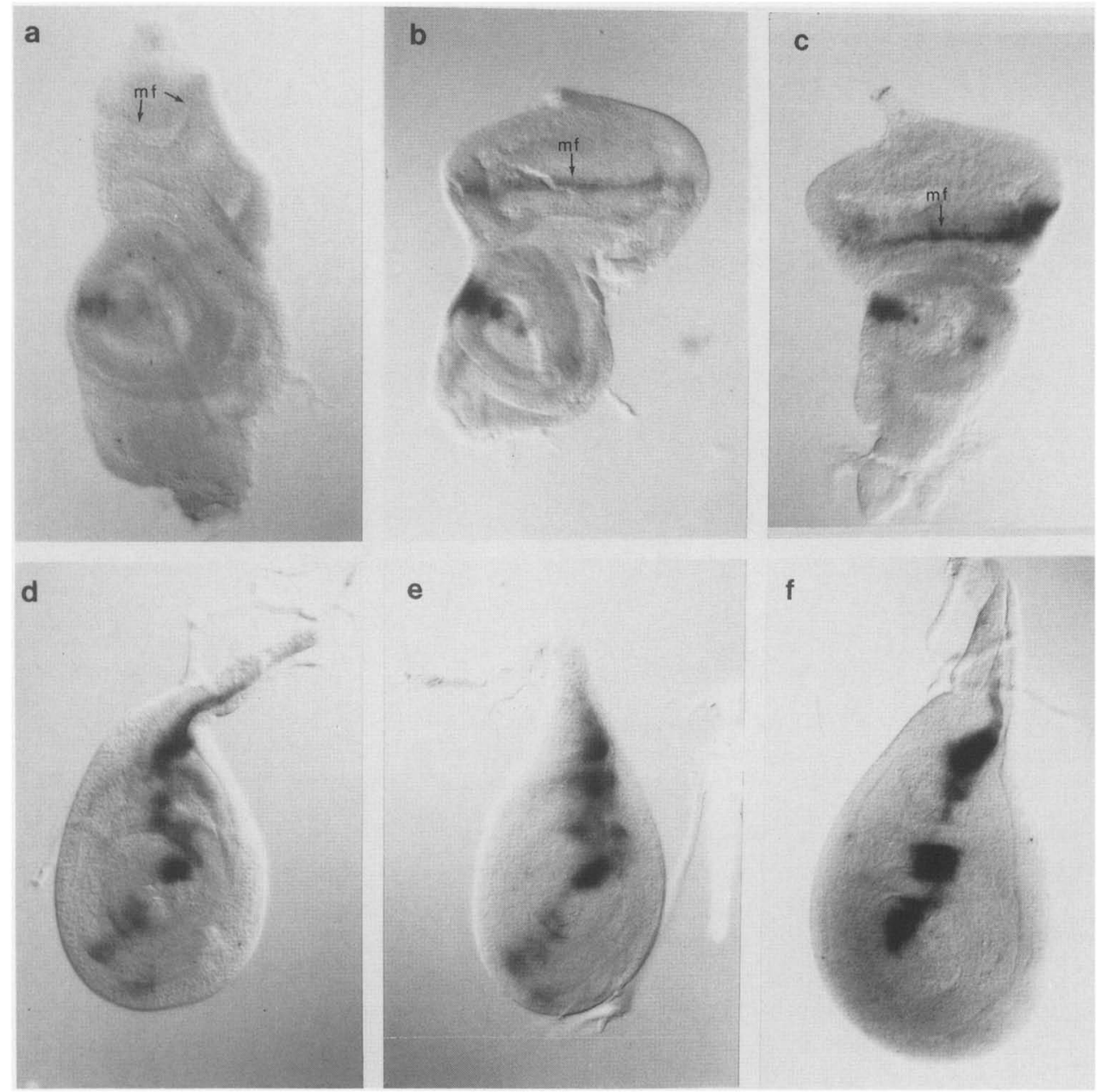

Figure 7. RNA expression in imaginal disks of $d p p^{d \cdot b l k}$ mutants and $d p p^{d \cdot b l k}$ mutants rescued by TnJMB7. Orientations are as in Fig. 1. Imaginal disks from $d p p^{d \cdot b l k}$ mutants (left) and $d p p^{d-b l k}$ mutants transgenic for two copies of TnJMB7 (center and right) were analyzed for expression of $d p p$ RNA (left and center) or adh RNA (right). (For wild-type expression of $d p p$, refer to Fig. 1.) No adh expression is detected in imaginal disks derived from larvae lacking the transposon (data not shown). (a, $d$ ) No $d p p$ expression is detectable in the morphogenic furrow (mf) of $d p p^{d-b l k}$ mutant eye disks, but antennal and leg disk expression is wild type. $(b, e)$ Two copies of TnJMB7 restore $d p p$ expression in the morphogenic furrow of $d p p^{d-b l k}$ mutant eye disks; the $d p p$ expression in the leg and antennal disks is the sum of the expression from the endogenous gene and the transposon. (c, $f$ ) TnIMB 7 drives $a d h$ expression in a subset of the positions normally expressing $d p p$.

with the presence of regulatory elements in the 106-110 fragment that are necessary for $d p p$ expression in the eye imaginal disk. No ectopic expression from TnJMB7 was detected (Fig. 7e).

\section{Rescue of dpp mutant phenotypes in other disks by TnIMB7}

TnJMB7 was crossed into $d p p$ mutant backgrounds exhibiting defects in almost all imaginal disk-derived structures. Chromosomal rearrangement breakpoints of the class III mutations lie $11-20 \mathrm{~kb} \mathrm{3}$ of the transcribed region (100-109 on the dpp genomic map; Fig. 8) and separate all of the regulatory sequences beyond the breakpoint from the dpp transcribed region. These alleles cause defects in all imaginal disk-derived portions of the adult cuticle; for example, the wing blade is re- duced to a stump (Fig. 9a). One copy of TnJMB7 provided sufficient $d p p$ expression to completely rescue the eye defects of the class III allele $d p p^{d 6}$ (not shown) and partially rescue leg, antennae, haltere, and wing (Fig. 9b) defects. Two copies of TnJMB7 allowed full phenotypic rescue of class III $\left(d p p^{d 6}\right)$ eye, leg, antennae, and haltere defects and substantial rescue of the wing defects as well (Fig. 9c). These animals are fertile and can be maintained as a stock. Class $\mathrm{V}$ mutations are chromosomal rearrangements that remove all of the $d p p^{d i s k}$ regulatory elements $>2 \mathrm{~kb} 3^{\prime}$ of the $d p p$-transcribed region. The mutant animals die as early pupae due to the absence of adult cuticle derived from the imaginal disks. With two copies of TnJMB7, 35-40\% of the mutant animals $\left(d p p^{d 12} / d p p^{d 14}\right)$ form a full adult cuticle, but most of these fail to eclose. Those that do eclose from the pupal case $(4-8 \%$ of the mutant progeny class) have normal 


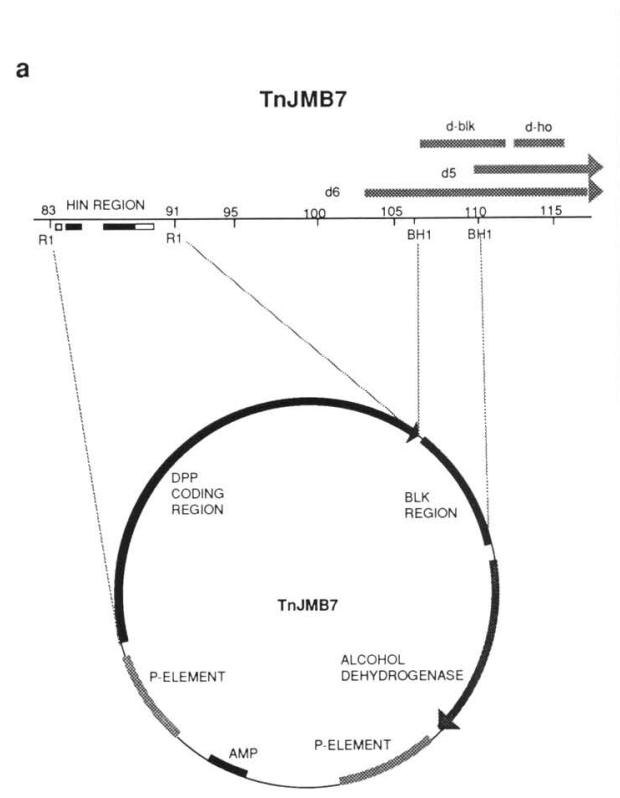

b

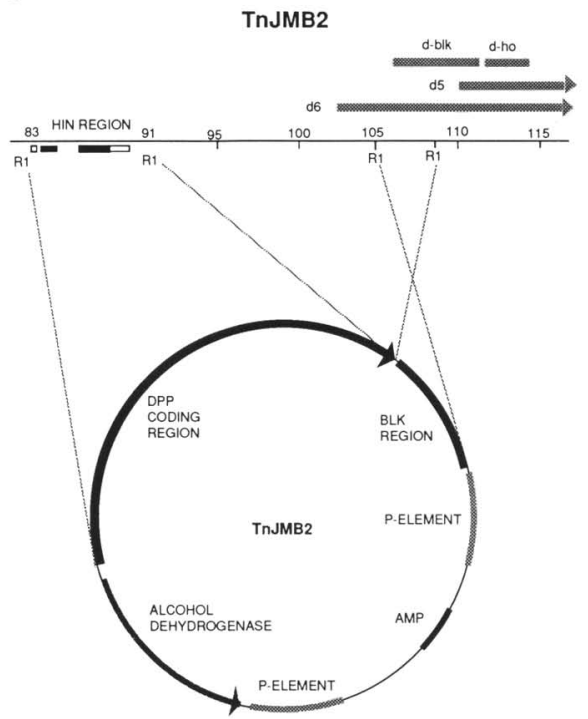

Figure 8. Maps of the transposons TnJMB7 $(a)$ and TnJMB2 $(b)$. The black line represents the genomic sequence, numbered in kilobases of cloned DNA on the $d p p$ chromosomal walk (St. Johnston et al. 1990). The shaded boxes above the line represent DNA removed by each of the indicated $d p p^{d i s k}$ mutations. $d p p^{d-b l k}$ and $d p p^{d-h o}$ are simple deletions. $d p p^{d 5}$ and $d p p^{d 6}$ are chromosomal rearrangements that remove additional DNA beyond the shaded region, as indicated by the arrowheads. Below the line, black boxes in the Hin region represent coding sequences for $d p p$; open boxes denote nontranslated exonic sequences. Each transposon contains an 8-kb EcoRI fragment from the Hin region, which consists of a promoter and the coding exons of the dpp gene, inserted into the P-element vector pPA1. TnJMB7 contains the 106-110 BamHI fragment placed at the $3^{\prime}$ end of the Hin region, in the same direction as the $d p p$ transcriptional unit. This fragment encompasses nearly $4 \mathrm{~kb}$ of the $5 \mathrm{~kb}$ deleted by the $d p p^{d-b l k}$ mutation. TnJMB2 contains the 105-109 EcoRI fragment placed at the $3^{\prime}$ end of the Hin region but in the opposite direction as the $d p p$ transcriptional unit. It should be noted, however, that the disk region fragments are both in the same orientation as the $a d h$ transcriptional unit in the two different plasmids. Arrows on the circular plasmid maps indicate the direction of transcription of $d p p$ and $a d h$. (R1) EcoRI; (BH1) BamHI,

eyes, some complete legs and some legs missing tarsal segments, and near full-sized, uninflated wings (not shown).

\section{Discussion}

Specific positions in imaginal disks correspond to specific positions and structures on the adult cuticle. The mechanisms by which a field of positional information is established and maintained in each imaginal disk are unknown. We have described the highly localized patterns of $d p p$ expression in the imaginal disks and established that the $3^{\prime}$-nontranscribed region of $d p p$ is necessary for proper expression in specific cells within imaginal disks. Our results indicate that $d p p$ expression is associated with the hypothetical proximal-to-distal axis of imaginal disk positional information. The mutant phenotypes of the $d p p^{d i s k}$ alleles also support a role for $d p p$ along the presumptive proximal-to-distal axis; however, it remains to be determined whether the role of $d p p$ is in establishing, maintaining, or transmitting imaginal disk positional information.

We have shown that $d p p$ mutations, which disrupt imaginal disk development, reduce the level or affect the pattern of $d p p$ expression in the imaginal disks. In the earliest disks examined from early third-larval instar, $d p p$ expression is already localized to specific regions of the disks; we suspect that $d p p$ may be localized even earlier and required throughout disk development. The pattern of $d p p$ expression in the leg and wing disks evolves as the disk cells proliferate during the thirdlarval instar and is maintained through the initial stages of disk eversion. The small size and altered morphology of imaginal disks caused by severe (class V) $d p p^{\text {disk }}$ mutations indicate that $d p p$ is required throughout disk development for cell proliferation and/or cell viability.

The earliest known event in establishing domains within imaginal disks is the formation of the anteriorposterior compartment boundary in the disks derived from the metameric regions of the embryo (for review, see Brower 1985). These cell lineage boundaries in the disks may correspond to the parasegment boundaries established at blastoderm when the disk primordia are set aside. Localized expression of $d p p$ transcripts is detected in head and thoracic segments of the embryo at positions corresponding to the imaginal disk primordia; this expression responds to segment polarity pattern formation genes that might be involved in localizing $d p p$ adjacent to the anterior-posterior compartment boundary (P.D. Jackson and F.M. Hoffmann, in prep.). Localization of $d p p$ expression relative to en reported here, and analysis of clones of $d p p$ mutant cells (Posakony et al. 1990) 

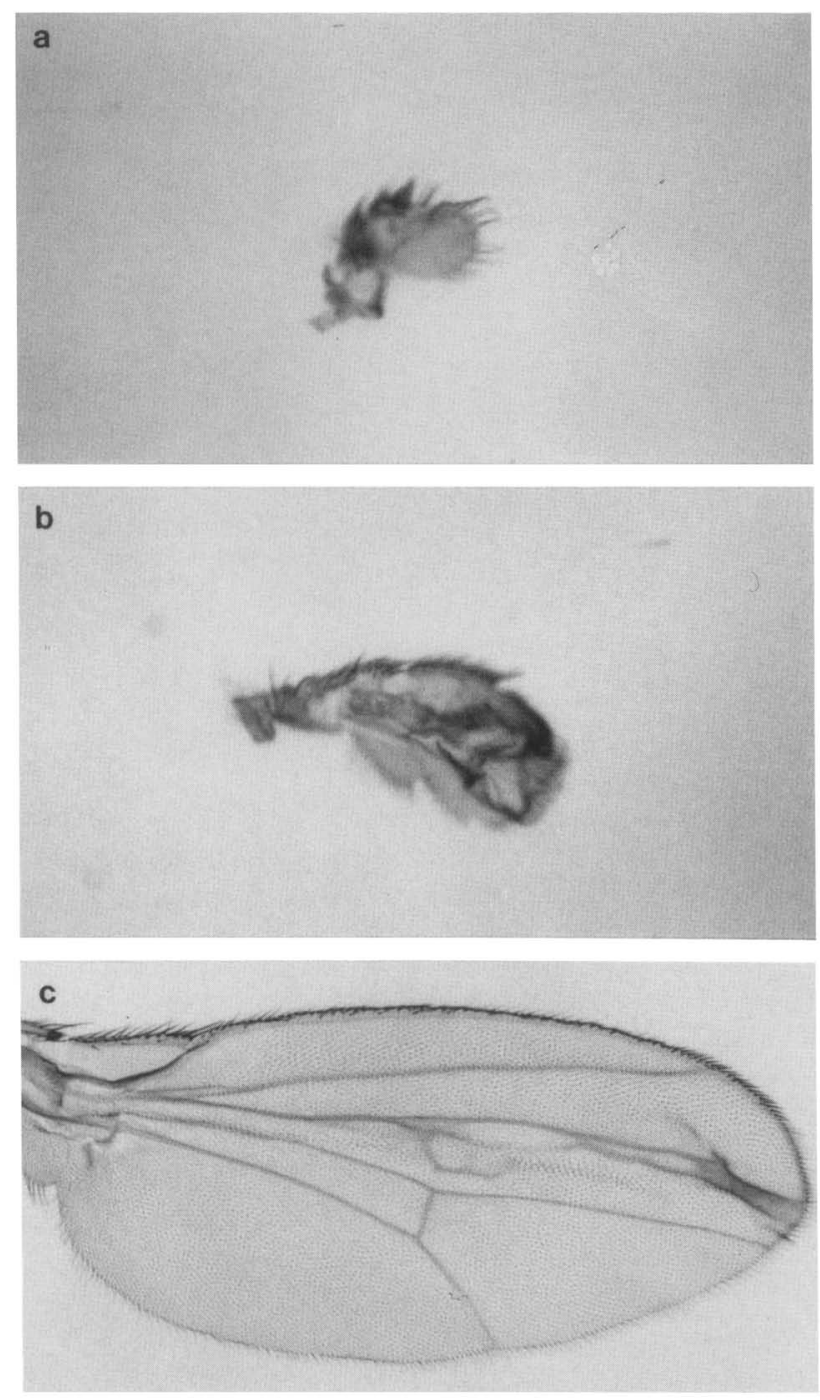

Figure 9. Progressive rescue of $d p p^{d 6}$ mutant wing phenotype with increasing copies of TnIMB7. Adult dorsal wing surfaces are shown at the same magnification. $(a) d p p^{d 6}$ class III mutants develop wing stumps that lack venation. $(b)$ One copy of TnJMB7 increases the size of $d p p^{d 6}$ mutant wings. (c) Two copies of TnJMB7 rescue the mutant wing to $80 \%$ wild-type size. Defects along the third longitudinal wing vein are observed in all genetic backgrounds homozygous for the transposon.

both support the conclusion that a band of cells immediately anterior to the compartment boundary express $d p p$. We believe that the compartment boundary may be used to orient $d p p$ expression along the presumptive proximal-to-distal axis of the wing and leg disks. The eye-antennal disk is derived from a nonmetameric portion of the embryo and does not exhibit the anteriorposterior cell lineage boundary until the third-larval instar (Morata and Lawrence 1978). Therefore, early expression in the eye-antennal disk of $d p p$ may be regulated by other positional cues.

Some portions of the $d p p^{d i s k}$ regulatory region are particularly potent in specific imaginal disks. This is illus- trated by the eye disk-specific defects of $d p p^{d-b l k}$, the wing disk-specific defects of $d p p^{d-h o}$, and the wing and eye disk-specific defects of $d p p^{d 5}$. The $d p p^{d-b l k}$ mutation specifically reduces the level of $d p p$ expression in the eye disk to below our limits of detection; either there must be some residual $d p p$ expression or $d p p$ expression prior to third instar in the $d p p^{d-b l k}$ eye disk because the $d p p^{d-b l k}$ mutant phenotype in the eye is not as severe as that caused by class III and class V $d p p$ mutations. The $d p p^{d-b l k}$ mutation does not cause any detectable change in the intensity or pattern of staining in disks other than the eye disk. Similarly, the $d p p^{d 5}$ mutation reduces the level of $d p p$ expression across the wing disk and somewhat in the eye disk but has no detectable effect on the level of expression in the leg disk, at least as deduced by the relative intensity of staining in RNA in situ hybridizations.

Although the regulatory elements affected by $d p p^{d-b l k}$ are extremely potent in the eye and the regulatory elements removed by $d p p^{d 5}$ are most critical in the wing disk, these two regions of $d p p$ regulatory elements are not disk-specific. Mutations like $d p p^{d 6}$ (for map positions of alleles, see Fig. 81, which remove the $d p p$ regulatory elements in 106-110 and the regulatory elements beyond position 110, cause severe defects in all imaginal disk-derived structures. In contrast, mutations like $d p p^{d 5}$, which remove only the regulatory elements beyond position 110 and leave the 106-110 region contiguous with the rest of the $d p p$, cause only moderate wing defects and mild eye defects. The presence of the regulatory elements in the $106-110$ region must provide dpp expression in all disks to account for the milder phenotype of $d p p^{d s}$. This is consistent with our observation that the 106-110 fragment drives adh expression in the leg and wing disks, as well as in the eye disk. Deletion of the elements on the 106-110 fragment by $d p p^{d-b l k}$, however, does not affect the development of disks other than the eye disk. This indicates that the regulatory elements beyond position 110, removed by both $d p p^{d 5}$ and $d p p^{d 6}$, but not by $d p p^{d-b l k}$, must also function in all disks. In support of this hypothesis, it has been reported that different disks use the same system of positional information (Bryant et al. 1978). Therefore, neither region is disk-specific, but each is likely to be position-specific. It is important to note, however, that specific regulatory regions, e.g., the 106-110 region, can be more or less critical to the development of specific disks. This is illustrated by the ability of one copy of TnJMB7, containing only the regulatory sequences in the 106-110 interval, to fully rescue the eye phenotype not only of $d p p^{d-b l k}$, but also of $d p p^{d 6}$. We conclude that the 106-110 regulatory region may not be eye disk-specific, but it is extremely potent in the eye disk.

Individual $d p p^{d i s k}$ region regulatory elements may respond to specific positions within a disk. Analysis of the $d p p^{d-b l k}$ phenotype indicates that deletion of part of the $d p p$ regulatory region specifically debilitates development in the ventral half of the eye disk, leading to ventrally localized cell death and the absence of ventrally oriented retinal cell clusters. Presumably, other $d p p$ reg- 
ulatory elements not affected by $d p p^{d-b l k}$ are active in the dorsal half of the eye disk. Similarly, the regulatory elements removed by the $d p p^{d-h o}$ mutation lead to a reduction in $d p p$ expression only near the center of the wing disk and the absence of an adult cuticular structure derived from the center of the disk. In the leg and antennal disks, some of the $d p p$ regulatory elements must discriminate between medial and lateral halves of the disks to provide much higher levels of expression in the lateral half.

Multiple regulatory regions in the $d p p^{\text {disk }}$ region are needed to provide the complete pattern of $d p p$ expression in any one disk, because individual regions appear to contribute only specific portions of the complete pattern. This is illustrated by position-specific reductions in expression caused by $d p p$ mutant alleles. Consistent with this, adh expression from TnJMB7 and TnJMB2 is in only a subset of the positions in the leg disk that express $d p p$. We predict that portions of the regulatory region on either side of the 106-110 fragment are responsible for the expression of $d p p$ in other parts of the disk.

The requirement for two copies of the $d p p$ gene for proper embryonic development indicates that embryonic cells require a certain level of $d p p$ expression (Irish and Gelbart 1987). Our observations indicate that imaginal disk cells are sensitive to the level of $d p p$ expression as well. For example, there is a significant improvement in the $d p p^{d 6}$ wing blade (Fig. $9 \mathrm{~b}, \mathrm{c}$ ) when the dose of TnJMB7 is increased from one copy to two copies. Two copies of TnJMB7 also improve the phenotype of $d p p^{d 12 /}$ $d p p^{d 14}$ (class V) mutant animals significantly more than one copy of TnJMB7. The $d p p^{d 12} / d p p^{d 14}$ mutant animals are not rescued as well as the $d p p^{d 6}$ (class III) mutant animals, because the class $\mathrm{V}$ alleles remove even more of the $d p p 3^{\prime}$ regulatory region. Perhaps three or four copies of TnJMB7 would provide more complete rescue of the class V phenotype. Apparently, the incomplete set of regulatory sequences on TnJMB7 can be partially compensated for by higher levels of $d p p$ expression in the wing disk achieved by two copies of the transposon. The compensatory effect of higher levels of $d p p$ expression in a subset of the cells that normally express $d p p$ may simply reflect the cell-nonautonomous property of the secreted $d p p$ protein. $d p p$ protein is secreted in S2 cells (Panganiban et al. 1990) and in embryos (G.E.F. Panganiban, R. Reuter, M.P. Scott, and F.M. Hoffman, in prep.); it is likely to be secreted in the imaginal disks as well. It is not known to what extent $d p p$ diffuses in imaginal disks, but the central location of its expression could allow most, if not all, of the cells of the disk to be exposed to the $d p p$ product, perhaps in a graded fashion. To more fully understand the function of $d p p$ in the disks, it will be important to determine the distribution of $d p p$ protein in the disk. The properties of the protein in culture (Panganiban et al. 1990b) and in the embryo (G.E.F. Panganiban, R. Reuter, M.P. Scott, and F.M. Hoffman, in prep.) lead us to predict that the secreted $d p p$ protein will be found in a fairly limited area due to association with other extracellular proteins.

We propose that specific disks and specific regions within a single imaginal disk may have different requirements for $d p p$ product, which are effectively met by an array of regulatory elements. These elements may differ in how well they function in different disks, either because they respond particularly well to disk-specific trans-acting factors or because they provide $d p p$ expression at a position in a disk where its function is particularly critical for the development of that disk. Further dissection of the $d p p^{d i s k}$ region should distinguish between these possibilities. It is not yet clear what the role of $d p p$ is in imaginal disk development and why its expression is so exquisitely localized. It will be important to determine whether localized expression of $d p p$ is essential to its role in disk development. We are particularly interested in the proteins that act on the $3^{\prime}$ cis-regulatory elements to cause expression of $d p p$ in these specific patterns. Identification of these molecules should bring us one step closer to understanding the molecular basis of positional information in the imaginal disks.

\section{Materials and methods}

Drosophila culturing

Drosophila stocks were grown on standard corn meal/sugar/ yeast media at $25^{\circ} \mathrm{C} . d p p^{d-h o}, d p p^{d-b l k}, d p p^{d 5}, d p p^{d 6}$, and $d p p^{d 14}$ are as described (Spencer et al. 1982; St. Johnston et al. 1990). All other stocks are described in Lindsley and Zimm (1985, 1990).

\section{Digoxigenin labeling of DNA probes for RNA in situ}

A modified method of Tautz and Pfeiffle (1989) was used to label the DNA fragments for whole-mount in situs. The $3-\mathrm{kb}$ EcoRI fragment or the 2-kb EcoRI/ApaI fragment of the $d p p$ cDNA E55 was used to detect $d p p$ mRNA. These fragments recognize the exons common to each transcript and were used interchangeably. The 4.5-kb EcoRI fragment from the genomic clone pen4.5R (from Dr. Allen Laughon, University of Wisconsin, Madison), which spans the first exon and the first intron, was used to detect the en transcripts. The $2.5-\mathrm{kb}$ Sall fragment of the transposon vector pPAl (Spradling 1986; gift of Dr. James Posakony, University of California, San Diego), which covers most of the $a d h$ gene fragment present in the vector, was used to detect the $a d h$ transcripts. Each fragment was digested in a volume of $100 \mu \mathrm{l}$ with HaeII and HaeIII to generate small fragments, phenol/chloroform-extracted, diluted to $2 \mathrm{ml}$ with $\mathrm{ddH}_{2} \mathrm{O}$, concentrated on a Centricon 10 column (Amicon) to 50 $\mu \mathrm{l}$, washed in $\mathrm{ddH}_{2} \mathrm{O}$ again, and concentrated again on a Centricon 10 column to $50 \mu \mathrm{l}$. The DNA concentration was determined by spectrophotometry. Approximately $500 \mathrm{ng}$ of DNA was boiled for $10 \mathrm{~min}$ in $10 \mu \mathrm{l} 1 \times$ Vogel buffer $95 \mathrm{mM}$ PIPES (pH 6.5), $5 \mathrm{~mm} \mathrm{MgCl}, 10 \mathrm{~mm} \beta$-mercaptoethanol), in the presence of $100 \mu \mathrm{g}$ pdN6 (Pharmacia), and placed on ice. The tube was spun briefly, and the following additions were made: $1 \mu \mathrm{l}$ $10 \times$ Vogel buffer, $2 \mu \mathrm{l} 10 \times$ nucleotide $\mathrm{mix}$ ( $1 \mathrm{mM}$ dATP, $1 \mathrm{~mm}$ dGTP, $1 \mathrm{~mm}$ dCTP, $0.65 \mathrm{~mm}$ dTTP, $0.35 \mathrm{~mm}$ 11-digoxigenin dUTP (Boehringer Mannheim Biochemicals) or Genius kit buffer 6 (Boehringer Mannheim Biochemicals), $1 \mu$ Klenow $(2 \mathrm{U} / \mu \mathrm{l})$, and $6 \mu \mathrm{H}_{2} \mathrm{O}$. After incubation at $16^{\circ} \mathrm{C}$ overnight, the reaction was boiled for $10 \mathrm{~min}$ and chilled on ice. One microliter of $10 \times$ Vogel buffer, $1 \mu \mathrm{l} 10 \times$ nucleotide mix, $1 \mu \mathrm{l}$ Klenow, and $7 \mu 1 \mathrm{H}_{2} \mathrm{O}$ were added, and the reaction was placed at $37^{\circ} \mathrm{C}$ for $4 \mathrm{hr}$. The reaction was stopped by adding $4 \mu \mathrm{l} 0.5 \mathrm{M}$ EDTA and heating to $80^{\circ} \mathrm{C}$ for $15 \mathrm{~min}$. Fifty micrograms of 
wheat germ tRNA and $2 \mathrm{ml}$ of PBT [PBS $(10 \mathrm{~mm} \mathrm{KPO}$ at $\mathrm{pH}$ $7.2,140 \mathrm{~mm} \mathrm{NaCl})+0.1 \%$ Tween 20 ] were added. The probes were concentrated to $50 \mu \mathrm{l}$ by using a Centricon 10 spin column, washed with $2 \mathrm{ml} \mathrm{PBT}$, and concentrated again to 50 $\mu \mathrm{l}$ by using a Centricon 10 column. The volume was adjusted with PBT to a probe concentration of $2 \mathrm{ng} / \mu \mathrm{l}$ (based on amount of template DNA added), and an equal volume of $100 \%$ deionized formamide was added for a final probe concentration of 1 $\mathrm{ng} / \mathrm{\mu l}$ in $50 \%$ formamide, $5 \mathrm{mM} \mathrm{KPO} 4(\mathrm{pH} 7.2), 70 \mathrm{mM} \mathrm{NaCl}$, and $0.05 \%$ Tween 20 .

\section{RNA in situ on whole-mount imaginal disks}

Some modifications were made to a protocol provided by Drs. W. Biggs and L. Zipursky (UCLA). Disks of various stages were dissected into PBS for 15 min or less to remove salivary glands, internal organs, and fat tissue (disks remained attached to mouth hooks and cuticle). Various stages of third-instar disks were dissected en masse and later staged by comparing to photographs in Bodenstein (1950). Everting disks were staged by picking white prepupae and allowing them to age 3.5 and $5.5 \mathrm{hr}$ at $25^{\circ} \mathrm{C}$ prior to dissection. The disks were then fixed for $15-20$ min in $4 \%$ paraformaldehyde in PBS on ice, fixed for 15-20 min in $4 \%$ paraformaldehyde, $0.6 \%$ Triton in PBS, at room temperature, washed three times for $5 \mathrm{~min}$ each in PBT, digested for 3-5 min with $10 \mu \mathrm{g} / \mathrm{ml}$ proteinase $\mathrm{K}$, washed two times for $5 \mathrm{~min}$ each in PBT plus $2 \mathrm{mg} / \mathrm{ml}$ glycine, washed three times for $5 \mathrm{~min}$ each with PBT, fixed $15 \mathrm{~min}$ in $4 \%$ paraformaldehyde $+0.2 \%$ gluteraldehyde in PBS at room temperature, washed five times for $5 \mathrm{~min}$ each in $\mathrm{PBT}$, washed $10 \mathrm{~min}$ in 50 : $50 \mathrm{mix}$ of $\mathrm{PBT} /$ hybridization buffer $150 \%$ deionized formamide, $5 \times$ SSC, $100 \mu \mathrm{g} / \mathrm{ml}$ sonicated, boiled salmon sperm DNA, $200 \mu \mathrm{g} / \mathrm{ml}$ wheat germ tRNA, $0.1 \%$ Tween 20 ) at room temperature, washed for $10 \mathrm{~min}$ in hybridization buffer at room temperature, and incubated in hybridization buffer at $48^{\circ} \mathrm{C}$ for at least 1 hr. Forty nanograms of heat-denatured digoxigenin-labeled probe was added to the prehybridizing disks such that the final volume was $\sim 100 \mu \mathrm{l}$. In the $d p p / e n$ double-labeling, $10 \mathrm{ng}$ of $d p p$ probe and $40 \mathrm{ng}$ of en probe were used. Hybridizations were allowed to continue for $36 \mathrm{hr}$ at $48^{\circ} \mathrm{C}$. Excess probe was washed off by a 20min wash in hybridization buffer, a 20-min wash in $50: 50 \mathrm{PBT} /$ hybridization buffer, and 10-12 hr of washes in PBT with about five changes of buffer. The tubes with the disks in PBT buffer were then placed on ice overnight. The disks were incubated for $1.5 \mathrm{hr}$ at room temperature with $1 \mathrm{ml}$ of a $1: 2000$ dilution of antidigoxigenin antibody (Genius kit) preabsorbed for $1 \mathrm{hr}$ to crushed, fixed larvae. The disks were then washed four times for 2 $\mathrm{hr}$ in PBT and washed for $15 \mathrm{~min}$ in staining buffer $100 \mathrm{mM}$ $\mathrm{NaCl}, 50 \mathrm{~mm} \mathrm{MgCl}, 100 \mathrm{~mm}$ Tris at $\mathrm{pH} 9.5,1 \mathrm{~mm}$ Levamisol, $0.1 \%$ Tween 20 ). The staining reaction was initiated by adding 1 $\mathrm{ml}$ staining buffer, $4.5 \mu \mathrm{l} \mathrm{NBT}$, and $3.5 \mu \mathrm{l} \mathrm{X}$-phosphate /Genius kit) and incubated for $2-6 \mathrm{hr}$ at room temperature. The reaction was stopped by several washes in PBT, and the disks were dissected and mounted in $80 \%$ glycerol.

\section{Acridine orange staining}

Some modifications were made to the protocol of Spreij (1971). Eye-antennal disks were dissected from wandering third-instar larvae in Drosophila Ringer's solution $(128 \mathrm{mM} \mathrm{NaCl}, 2 \mathrm{mM}$ $\mathrm{KCl}, 35.5 \mathrm{~mm}$ sucrose, $5 \mathrm{~mm}$ HEPES, $1.8 \mathrm{~mm} \mathrm{CaCl}, 4 \mathrm{~mm}$ $\mathrm{MgCl}_{2}$ at $\left.\mathrm{pH} 7.1\right)$ and stained with $1.6 \times 10^{-6} \mathrm{M}$ acridine orange (Aldrich) in Ringer's solution for $5 \mathrm{~min}$. After washing three times briefly in Ringer's solution, the disks were mounted in Ringer's solution and viewed immediately by fluorescence microscopy.
Creating transgenic fly lines with JMB2 and JMB7

Transgenic fly lines were created by the method of Spradling (1986). TnJMB2 and TnJMB7 were made in the P-element vector $\mathrm{pPAl}$, which contains the adh gene as a selectable marker. The defective P-element $\Delta 2,3$ (Robertson et al. 1988) was used to integrate the transposons. TnJMB7 is inserted on the second chromosome, and TnJMB2 is inserted onto TM2.

\section{Transposon rescue of dpp mutant phenotypes}

TnJMB7 was recombined onto the following $d p p$ mutant chromosomes: $d p p^{d-b l k} a d h^{f n 6}$ pr $c n, d p p^{6} a d h^{f n 6}$ pr cn, $d p p^{12} a d h^{f n 6}$ pr $\mathrm{cn}$, and $d p p^{14} a d h^{f n 6}$ pr cn. Isogenic recombinant chromosomes were balanced over $\mathrm{CyO}^{\text {nb }}$ and tested for rescue of $d p p$ mutant phenotypes.

\section{Sections of Drosophila eyes}

Drosophila eyes were prepared for sectioning as described by Ready et al. (1976). Sections were cut on a Reichert-Jung ultramicrotome and stained with toluidine blue for light microscopy.

\section{Scanning electron microscopy}

Adult Drosophila were dissected mid-thorax, rinsed in PBS, and fixed in $2.5 \%$ glutaraldehyde, $2 \%$ paraformaldehyde in $0.1 \%$ $\mathrm{NaPO}_{4}(\mathrm{pH} 7.0)$ for $30 \mathrm{~min}$ to overnight at $4^{\circ} \mathrm{C}$. The samples were rinsed three times in $0.1 \mathrm{M} \mathrm{NaPO}_{4}$ and successively dehydrated in ethanol steps of $35 \%, 50 \%, 70 \%$, and $85 \%$, two steps of $95 \%$, and three steps of $100 \%$ for 5 min each. Samples were dehydrated further with liquid $\mathrm{CO}_{2}$ in a Tousimis Samdri-780A critical point drier, mounted onto studs with conducting silver paint, allowed to air-dry for $10-20 \mathrm{~min}$, and sputter-coated with gold. Scanning electron micrographs were generated on a Hitachi S/570 microscope.

\section{Bright-field microscopy}

Dissected appendages from adult Drosophila were mounted in Canada balsam/methyl salicylate $(6 \mathrm{~g} / 4 \mathrm{ml})$ and viewed for light microscopy.

\section{Acknowledgments}

Support for this research was provided by grants from the American Cancer Society to F.M.H., Cancer Center Core Support CA07175 from the National Cancer Institute (NCI) to H.C. Pitot, National Institutes of Health training grant GM07215 to J.D.M., and NCI training grant T32-CA09135 to R.J.M. We thank Dr. Allen Laughon, Dr. John S. Doctor, Grace Panganiban, and Frank Gertler for their perceptive comments on the manuscript. We thank Melissa Curtis for providing expertise with the scanning electron microscopy, and Dr. Carol A. Sattler and Pei Sue Zhang for eye sections.

The publication costs of this article were defrayed in part by payment of page charges. This article must therefore be hereby marked "advertisement" in accordance with 18 USC section 1734 solely to indicate this fact.

\section{References}

Baker, N.E. 1988. Transcription of the segment polarity gene wingless in the imaginal disks of Drosophila, and the phenotype of a pupal-lethal wg mutation. Development 102: 489497. 
Blackman, R.K., R. Grimaila, M.D. Koehler, and W.M. Gelbart. 1987. Mobilization of hobo elements residing within the decapentaplegic gene complex: Suggestion of a new hybrid dysgenesis system in Drosophila melanogaster. Cell 49: 492-505.

Bodenstein, D. 1950. The postembryonic development of Drosophila. In Biology of Drosophila (ed. M. Demerec), pp. 275-368. John Wiley, New York.

Brower, D.L. 1985. The sequential compartmentalization of Drosophila segments revisited. Cell 41: 361-364.

Brower, D.L., M. Wilcox, M. Piovant, R.J. Smith, and L.A. Reger. 1984. Related cell-surface antigens expressed with positional specificity in Drosophila imaginal discs. Proc. Natl. Acad. Sci. 81: 7485-7489.

Bryant, P.J. 1978. Pattern formation in imaginal discs. In The genetics and biology of Drosophila (ed. M. Ashburner and T.R.F. Wright), pp. 229-335. Academic Press, London.

- 1988. Localized cell death caused by mutations in a Drosophila gene coding for a transforming growth factor- $\beta$ homolog. Dev. Biol. 128: 386-395.

Bryant, P.J., P.N. Adler, C. Duranceau, M.J. Fain, S. Glen, B. Hsei, A.A. James, C.L. Littlefield, C.A. Reinhard, S. Straus, and H.A. Schneiderman. 1978. Regulative interactions between cells from different imaginal disks of Drosophila melanogaster. Science 201: 928-930.

Fristrom, D. 1976. The mechanism of evagination of imaginal discs of Drosophila melanogaster. Dev. Biol. 54: 163-171.

Gelbart, W.M. 1989. The decapentaplegic gene: A TGF- $\beta$ homologue controlling pattern formation in Drosophila. Development (Suppl.) 107: 65-74.

Hoffmann, F.M. 1990. Developmental functions of decapentaplegic, a member of the TGF- $\beta$ family in Drosophila. In $G e$ netics of pattern formation and growth control (ed. A.P. Mahowald), pp. 103-124. Wiley-Liss, New York.

Hoffmann, F.M. and W. Goodman. 1987. Identification in transgenic animals of the Drosophila decapentaplegic sequences required for embryonic dorsal pattern formation. Genes Dev. 1: 615-625.

Irish, V.I. and W.M. Gelbart. 1987. The decapentaplegic gene is required for dorsal-ventral patterning of the Drosophila embryo. Genes Dev. 1: 868-879.

Kornberg, T., I. Siden, P. O'Farrell, and M. Simon. 1985. The engrailed locus of Drosophila: In situ localization of transcripts reveals compartment-specific expression. Cell 40: $45-53$.

Lindsley, D.L. and G. Zimm. 1985. The genome of Drosophila melanogaster, Part 1: Genes A-K. Dros. Inf. Serv. 65.

- 1985. The genome of Drosophila melanogaster, Part 4: Genes L-Z. Dros. Inf. Serv. 68.

Meinhardt, H. 1982. Models of biological pattern formation. Academic Press, London.

Morata, G. and P. Lawrence. 1978. Anterior and posterior compartments in the head of Drosophila. Nature 274: 473-474.

Padgett, R.W., R.D. St. Johnston, and W.M. Gelbart. 1987. A transcript from a Drosophila pattern gene predicts a protein homologous to the transforming growth factor- $\beta$ family. Nature 325: 81-84.

Panganiban, G.E.F., K.E. Rashka, M.D. Neitzel, and F.M. Hoffmann. 1990. Biochemical characterization of the Drosophila $d p p$ protein, a member of the transforming growth factor- $\beta$ family of growth factors. Mol. Cell. Biol. 10: 2669-2677.

Posakony, L. 1987. The role of the decapentaplegic gene complex in the development of imaginal discs in Drosophila melanogaster. Ph.D. dissertation. Harvard University, Cambridge, MA.

Posakony, L.G., L.A. Raftery, and W.M. Gelbart. 1990. Wing formation in Drosophila melanogaster requires decapentaplegic gene function along the anterior-posterior compartment boundary. Mech. Dev. (in press).

Postlethwait, J.H. 1978. Clonal analysis of Drosophila cuticular patterns. In The genetics and biology of Drosophila (ed. M. Ashburner and T.R.F. Wright), pp. 359-441. Academic Press, London.

Ready, D.F., T.E. Hanson, and S. Benzer. 1976. Development of the Drosophila retina, a neurocrystalline lattice. Dev. Biol. 53: 217-240.

Robertson, H.M., C.R. Preston, R.W. Phillis, D.M. JohnsonSchlitz, W.K. Benz, and W.R. Engels. 1988. A stable source of P-element transposase in Drosophila melanogaster. Genetics 118: 461-470.

Spencer, F.A. 1984. The decapentaplegic gene complex and adult pattern formation in Drosophila. Ph.D. dissertation, Harvard University, Cambridge, MA.

Spencer, F.A., F.M. Hoffmann, and W.M. Gelbart. 1982. Decapentaplegic: A gene complex affecting morphogenesis in Drosophila melanogaster. Cell 28: 451-461.

Spradling, A.C. 1986. P element-mediated transformation. In Drosophila: A practical approach (ed. D.B. Roberts), pp. 175-198. IRL Press, Oxford.

Spreij, T.H. 1971. Cell death during the development of the imaginal disks of Calliphora erythrocephala. Neth. J. Zool. 21(3): $221-261$.

St. Johnston, R.D., F.M. Hoffmann, R.K. Blackman, D. Segal, R. Grimaila, R.W. Padgett, H.A. Irick, and W.M. Gelbart. 1990. The molecular organization of the decapentaplegic gene in Drosophila melanogaster. Genes Dev. 4: 1114-1127.

Tautz, D. and C. Pfeiffle. 1989. A non-radioactive in situ hybridization method for the localization of specific RNAs in Drosophila embryos reveals translational control of the segmentation gene hunchback. Chromosoma 98: 81-85.

White, R.A.H. and M. Wilcox. 1985. Distribution of Ultrabithorax proteins in Drosophila. EMBO J. 4: 2035-2043.

Wieschaus, E. and W. Gehring. 1976. Clonal analysis of primordial disc cells in the early embryo of Drosophila melanogaster. Dev. Biol. 50: 249-263.

Wirz, J., L.I. Fessler, and W.J. Gehring. 1986. Localization of the Antennapedia protein in Drosophila embryos and imaginal discs. EMBO J. 5: 3327-3334. 


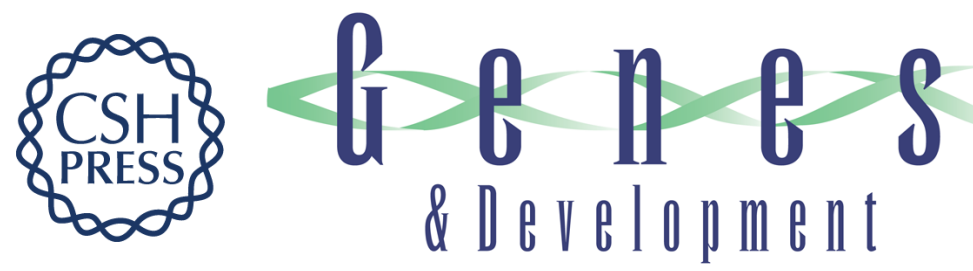

\section{Pattern-specific expression of the Drosophila decapentaplegic gene in imaginal disks is regulated by 3' cis-regulatory elements.}

J D Masucci, R J Miltenberger and F M Hoffmann

Genes Dev. 1990, 4:

Access the most recent version at doi:10.1101/gad.4.11.2011

References This article cites 23 articles, 9 of which can be accessed free at:

http://genesdev.cshlp.org/content/4/11/2011.full.html\#ref-list-1

License

Email Alerting

Service

Receive free email alerts when new articles cite this article - sign up in the box at the top right corner of the article or click here.

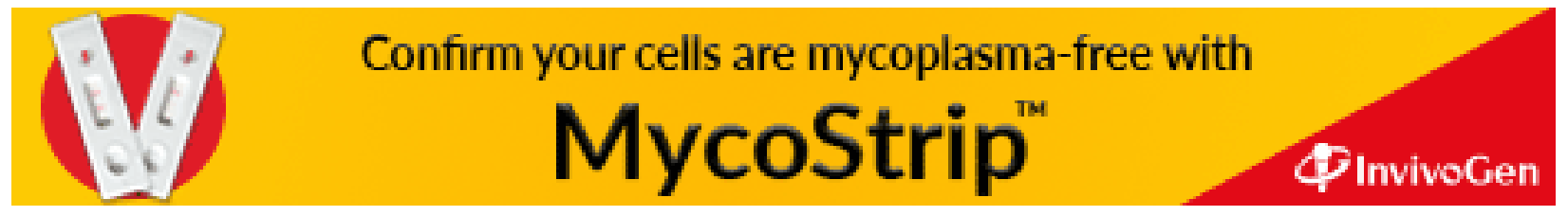

